\title{
Study on the relationship between menopausal age, body mass index and total number of teeth
}

\author{
Ioana Madalina Lescai ${ }^{1,2}$, Laurenta Lelia Mihai ${ }^{3}$, Monica Mihaela Cirstoiiu ${ }^{4,5}$ \\ ${ }^{1}$ Doctoral School, "Carol Davila" University of Medicine and Pharmacy, Bucharest, Romania \\ 2Blident Help Dental Clinic, Bucharest, Romania \\ ${ }^{3}$ Department of Periodontology and Oral Pathology, "Titu Maiorescu“ University, Bucharest, Romania \\ 4"Carol Davila" University of Medicine and Pharmacy, Bucharest, Romania \\ ${ }^{5}$ Department of Obstetrics \& Gynaecology, University Emergency Hospital, Bucharest, Romania
}

\begin{abstract}
Objectives. The objective of this study is to assess the state of oral health, from the perspective of the number of teeth present, according to the body mass index as a representative element of health.

Materials and methods. This study consists of 180 patients from a private dental practice. All 180 patient records were reviewed for an assessment of the number of teeth present and the body mass index at menopausal age. The cohort of patients was divided into two groups, a group of women aged 40 to 54.9 years and the second group, which included women aged 55 and over. The 2 groups were divided based on premenopausal (group I) and postmenopausal (group II) age.

Results. The average number of present teeth (based on radiographs) for the entire population of 180 patients is 22.02 teeth. In group I, for the 109 women in the premenopausal group, the average number of teeth is 23.94 . The total number of teeth for the 71 women in the postmenopausal group is 19.07. The average BMI for the entire group of patients is $27.24 \mathrm{~kg} / \mathrm{m} 2$, with a lower value in group I, $26.25 \mathrm{~kg} / \mathrm{m} 2$, compared to group II, $28.78 \mathrm{~kg} / \mathrm{m} 2$.

Discussions. The average number of teeth in the premenopausal group is higher than in the postmenopausal group. The average body mass index places the whole series of patients in the overweight category.

Conclusions. This study shows that the relationship between BMI and the number of lost teeth is positive and proportional, BMI increases with increasing number of teeth lost at premenopausal age.
\end{abstract}

Keywords: premenopause, menopause, dental status, body mass index, age

\section{INTRODUCTION}

Menopause is caused by permanent alterations in the ovaries' hormonal and reproductive functions. Natural menopause is a normal stage in a woman's life, described as the cessation of natural menstruation for 12 months in a row at the age of 45 to 55 [1]. Hormonal alterations have an impact on a woman's reproductive system as well as other aspects of her life. Hormones have a significant impact on the quality of life and the systemic bone loss. During menopause the gums become more vulnerable to plaque, increasing the risk of gingivitis and advanced periodontitis [2,3].
Skeletal bone might be an endocrine target tissue. Low bone density is believed to be linked to a high body mass index [4-6]. Not only are postmenopausal women at risk for skeletal loss, but also for alveolar bone loss from periodontitis, which can lead to tooth loss [7-9]. Tooth loss occurs when a tooth is separated from its supporting structure, due tooth decay, periodontal disease, periapical infection, endodontic therapy failure or fractures $[10,11]$.

The assessment of oral status can also be performed according to the body mass index-BMI. The objective of this study is to make an assessment of oral health, from the perspective of the 
number of teeth present, based on the body mass index as a representative element of health. All these were be evaluated according to the age of the patients, who will be classified into two distinct groups, premenopause and menopause. In this way, the evaluation of the dental status can potentially be performed according to or in correlation with the body mass index.

\section{AIM}

The aim of this study was to investigate whether a correlation exists between body mass index and the number of teeth in menopausal women.

\section{MATERIALS AND METHODS}

We conducted a retrospective study and analized the data from the medical charts of 180 patients aged between 40 and 84 years that presented to the Blident Help Dental Clinic, Bucharest, for specific consultations and treatments between April 2018 and June 2020. Data analysis and processing was completed with the help of SPSS 20.0 program.

Before the study began, informed consent was obtained. The confidentiality of all records, documents, X-rays, and case-report forms was maintained. Personal information, such as a person's name or any data that could have been identified, were not extracted in order to protect the information's confidentiality. Only the principal investigator and coinvestigators had access to the records. As the study was retrospectively, it did not require the opinion of the Ethics Committee of „Carol Davila" University of Medicine and Pharmacy.

Based on the radiographic evidence, an evaluation was performed in terms of the number of teeth for each patient. Practically, the total number of teeth present at the time of the consultation was determined. There are also separation criteria that have been taken into account and these refer to the fact that if dental fragments are present on the arch, the respective dental position will appear as missing (zero) tooth. Thus, the irretrievable root fragments were not considered as teeth present. These situations involve local extraction and treatment, possibly prosthetic, implant, and were not included in the number of teeth. In patients with canines or premolars included, only teeth that, according to the treatment plan accepted by the patients, were to be orthodontically pulled on the arch were considered. The lack of agreement for an orthodontic treatment, in this case, led to the consideration of those included teeth as absent. Regarding the molars 3, if they are present radiologically but are not erupted on the arch due to the lack of any masticatory function, they are considered absent. The dental implant is considered a missing tooth. In total, intact and viable teeth, intact teeth with endodontic treatment, restored coronary or corono-radicular teeth, fractured teeth recoverable from a prosthetic point of view were considered present. Thus, for each patient a primary correlation was made between the oral clinical examination and the evaluation of the panoramic radiography. For each patient, the first panoramic radiograph was taken into account, even if later, in the therapeutic dynamics, it needed additional investigations. Each patient is included in the study with a single visit. Also, during the consultation of each patient, anamnestic data on height and weight were taken at the time of radiological examination, so as to eliminate variations at least between the time of examination and visits before or after the radiological examination. Height and weight are used to make the body mass index according to the formula: the body mass index is equal to the ratio between the weight expressed in kilograms and the square of the height expressed in meters.

\section{RESULTS}

The 180 women were divided into 2 groups according to age at the time of presentation. The first group consists of women between 40 and 54.9 years and in the second group are included women over 55 years. The age limit, 55 years, is chosen in accordance with the population situation in Romania, being the age limit for premenopausal and menopausal conditions. Of the 180 women, 109 (60.5\%) are from group 1, premenopause, 40-54.9 years. In group 2, postmenopause, over 55 years are 71 women $(39.5 \%)$.

All women in the 2 groups benefited from a dental consultation and a panoramic x-ray to record dental status.

For simplification, the average number of teeth present was made for the entire age range of the two groups but also for each group. For each pa- 
tient, however, from each of the 2 groups an exact situation was made for each dental position.

For the entire number of patients, 180, the average number of teeth present radiologically is 22.02 teeth and for each group the situation is as follows: for the 109 women in group 1, 40-54 years, the average number of teeth is 23.94 . For the 71 women in group 2, over 55 years old, the average number of teeth is 19.07 .

The total number of patients was 180 aged between 40 and 84 years (figure 1). The average age is 54 years. Interpretations can be made on both the whole series of patients but the importance of this study is within and between the two premenopausal and postmenopausal groups. The premenopausal group called group I comprises patients aged between 40 and 54.9 years. The postmeno- pausal group, called group II, includes patients aged 55 to 84 years. The mean age in group I is 47.79 years out of a number of 109 patients. Group II has a mean age of 64.72 out of a total of 71 patients. The average number of teeth for the entire series of subjects is 22.02 with a range between 2 and 32 teeth. The average number of teeth for group I is 23.94 with a range between 7 and 32 teeth. The average number of teeth for group II is 19.07 with a range between 2 and 30 teeth. The body mass index for the entire series of patients is $27.24 \mathrm{~kg} / \mathrm{m}^{2}$ with a range of $18.2 \mathrm{~kg} / \mathrm{m}^{2}$ and 42.2 $\mathrm{kg} / \mathrm{m}^{2}$. For group I the body mass index is 26.25 $\mathrm{kg} / \mathrm{m}^{2}$ with a range of $18.6-42.2 \mathrm{~kg} / \mathrm{m}^{2}$. The body mass index for group II is $28.78 \mathrm{~kg} / \mathrm{m} 2$ with a range of $18.2-40.4 \mathrm{~kg} / \mathrm{m}^{2}$ (Table 1 , Table 2).
TABLE 1. Group I average BMI and number of teeth by age

\begin{tabular}{|l|r|r|}
\hline Group I Age & Average BMI & $\begin{array}{l}\text { Average number of } \\
\text { teeth }\end{array}$ \\
\hline 40 & 26.49 & 28.25 \\
\hline 41 & 24.22 & 30.00 \\
\hline 42 & 21.88 & 22.00 \\
\hline 43 & 23.97 & 27.33 \\
\hline 44 & 31.76 & 24.00 \\
\hline 45 & 26.14 & 24.00 \\
\hline 46 & 24.00 & 25.20 \\
\hline 47 & 29.03 & 23.30 \\
\hline 48 & 26.44 & 24.00 \\
\hline 49 & 26.43 & 24.29 \\
\hline 50 & 26.34 & 23.00 \\
\hline 51 & 22.46 & 24.00 \\
\hline 52 & 25.29 & 19.67 \\
\hline 53 & 25.94 & 23.14 \\
\hline 54 & 28.05 & 19.17 \\
\hline
\end{tabular}

TABLE 2. Group II average BMI and number of teeth by age

\begin{tabular}{|c|c|c|}
\hline Group II Age & Average BMI & $\begin{array}{l}\text { Average number of } \\
\text { teeth }\end{array}$ \\
\hline 55 & 30.74 & 23.20 \\
\hline 56 & 29.23 & 26.67 \\
\hline 57 & 25.60 & 17.00 \\
\hline 58 & 26.98 & 18.00 \\
\hline 59 & 25.25 & 15.00 \\
\hline 60 & 27.76 & 22.33 \\
\hline 61 & 29.19 & 20.57 \\
\hline 62 & 25.61 & 20.00 \\
\hline 63 & 26.56 & 23.25 \\
\hline 64 & 30.82 & 19.00 \\
\hline 65 & 29.84 & 10.50 \\
\hline 66 & 31.07 & 22.00 \\
\hline 67 & 29.83 & 20.75 \\
\hline 68 & 37.04 & 9.00 \\
\hline 69 & 26.34 & 16.40 \\
\hline 70 & 27.85 & 15.67 \\
\hline 71 & 31.12 & 18.67 \\
\hline 72 & 40.40 & 7.00 \\
\hline 73 & 29.14 & 17.00 \\
\hline 74 & 26.16 & 12.00 \\
\hline 75 & 27.11 & 24.00 \\
\hline 76 & 28.52 & 28.00 \\
\hline 77 & 28.89 & 18.00 \\
\hline 81 & 27.34 & 18.00 \\
\hline 83 & 20.70 & 14.00 \\
\hline 84 & 26.72 & 22.00 \\
\hline
\end{tabular}




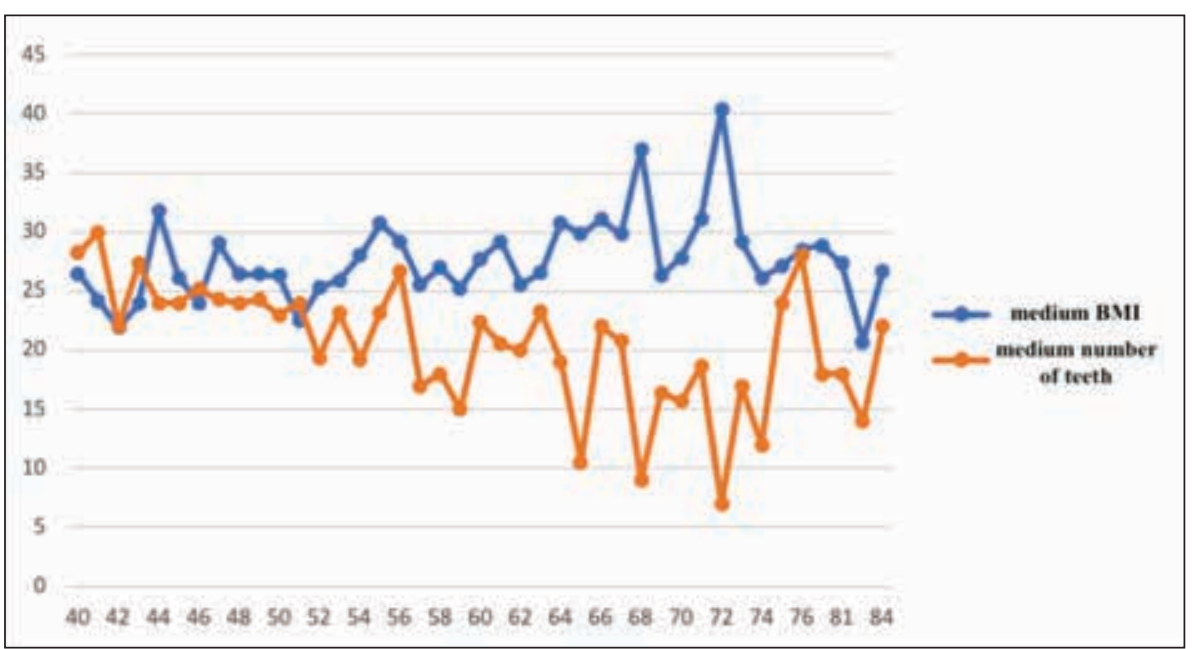

FIGURE 1. Number of teeth by age and BMI for all patients

Regarding the number of teeth for the entire series of patients, the average number of teeth was 22.02 teeth. The range obtained for the entire series of patients was 2 to 32 teeth. The average number of teeth for group I, premenopause, was 23.94 with a range of 7-32. The average number of teeth for group II, postmenopause was 19.07 teeth with a range of 2 to 30 teeth.

The average body mass index value, for the entire series of 180 patients, is 27.24 with a range between 18.2 and 42.2. For group I, premenopause, the average value of BMI is $26.25 \mathrm{~kg} / \mathrm{m}^{2}$ with a range of $18.6-42.2 \mathrm{~kg} / \mathrm{m}^{2}$. For group II, postmenopausal, the average value of BMI is 28.78 with a range between $18.2-40.4 \mathrm{~kg} / \mathrm{m}^{2}$ (Table 3).

TABLE 3. Average age. Number of teeth and BMI for both groups

\begin{tabular}{|l|c|c|c|}
\hline & Group I & Group II & All patients \\
\hline Age & $40-54.9$ & $>55$ & $40-84$ \\
\hline Average age & 47.79 & 64.72 & 54 \\
\hline Average number of teeth & 23.94 & 19.07 & 22.02 \\
\hline Average BMI & 26.25 & 28.78 & 27.24 \\
\hline
\end{tabular}

\section{DISCUSSION}

One conclusion of the study is represented by the homogeneous distribution of patients by age ranges of the 2 groups, premenopause and postmenopause. This is not the result of any manipulation of data or selection of patients but is a reflection of the actual patient of the dental clinic located for this study. This is an advantage in terms of interpreting the data obtained as a real picture of the actual clinical situation.
It is interesting to note that the average number of teeth 22.02 for the whole series of women is lower than the average number of teeth in group I (24 teeth) and higher than the average number of teeth in group II, ie 19. As a result, it is possible to say that statistical evidence shows that the premenopausal group has more teeth on average than the postmenopausal group. Interestingly, however, there are people within group II who approach the maximum anatomical limit of 32 teeth, but the minimum limit is significantly different from that of group I. However, the extremes of both groups are poorly represented numerologically, their average being in fact representative.

It should be noted that the average value of BMI in group I is lower than the average value in group II but both are above 25, the lower limit for the overweight population according to the World Health Organization. Basically, by performing the average, the entire series of patients is placed in the overweight category. It is noteworthy that the variation between the average BMI of group I and group II is $7.14 \%$ in favor of group II.

The variation of average number of teeth is $20.34 \%$ in favor of group I. A purely numerological primary observation concludes that a $7 \%$ increase in BMI would lead to the loss of $20 \%$ of teeth. It would mean that in group II, the BMI index increases compared to group I, while the number of teeth decreases. This denotes an inversely proportional relationship. However, caution is needed in interpreting these data because it is anticipated that, with increasing age, the BMI will lose its relevance in terms of oral health. 
Given the number of teeth for each group, in the case of patients over 65 years with less than 20 remaining teeth, in this study there are 22 patients (30.99\% of Group II) who, in theory, would be conclusions of Hasiao-Yun $\mathrm{Hu}$ (2015) [12] in danger of death in the next 5 years regardless of medical cause. This would mean that given the data of the National Institute of Statistics which reports the age of female mortality around 72 years, it means that, theoretically, these 30 patients are approaching the age of 70 which is itself near the average age of death in Romania for the female population.

Compared to the 2010 Buffalo Osteo Perio study [13], the average BMI in this study is 27.24, compared to 30 in the American study. The more positive placement of the population in this study compared to the American population is debatable, although the difference is in the total number of followed patients - 180 compared to 1,341 .

Considering Oikarinen's 2013 study [14], the correlation of BMI with the number of teeth is low in the 60-90 age group. This may have a major implication because in this study, the BMI variation of $7 \%$ between the 2 groups is low, while the number of teeth continues to decrease. As a result, it is prudent to consider in the analysis of the results of this study that within the 55-84 age group from the age of 60 the influence of BMI would be minimal and only lead to leveling or weighting the correlation with the number of teeth for patients under this age.

It is observed that for the age group 55-60 years, which includes 20 patients $(28.17 \%$ of the total group II), the correlation between BMI and the number of teeth is a positive, directly proportional, BMI increases with increasing number of teeth. While in the age range 60 to 84 years the relationship between BMI and the number of teeth is negative, inversely proportional, the major disparities being located at 65,68 and 72 years, with a number of less than 10 teeth for BMI over $30 \mathrm{~kg} / \mathrm{m}^{2}$. It follows that in the present study, obesity certified by a BMI $>30 \mathrm{~kg} / \mathrm{m}^{2}$ is associated with a small number of teeth, below 10 , with a potential theoretical risk of mortality in the next 5 years of life. This correlation is conflicting given some studies [14] that suggested that BMI was irrelevant after the age of 60 . However, the small number of patients in this age range of this study, $\mathrm{N}=20$, requires caution in interpreting the data. What is relevant, however, is that for the 55-60 age group, the correlation between BMI and the number of teeth is inverse to that of the 61-84 age subgroup. In the 55-60 age range, the average BMI is over 25 , corresponding to the overweight category. The maximum number of teeth 26.66 corresponds to a BMI of $29.22 \mathrm{~kg} / \mathrm{m}^{2}$. The minimum average values are obtained at the age of 59 with a number of 15 teeth and a BMI of $25 \mathrm{~kg} / \mathrm{m}^{2}$.

The study shows that between 56 and 59 years of age BMI correlates positively with the number of teeth, both values having a decreasing trend, but BMI does not fall below $25 \mathrm{~kg} / \mathrm{m} 2$ and the number of teeth does not fall below 15.56 and 59 years old show a tendency towards normal weight, tending to remain with an average number of 15 teeth.

Between 40 and 54 years, the relationship between BMI and the number of teeth is linear with several points of divergence, the most pronounced being for the age category 44 years when a BMI of over $30 \mathrm{~kg} / \mathrm{m}^{2}$ is observed, as such obesity, with an average number of teeth of 24 . However, there are only 4 patients at this benchmark (3.64\%). Between 46 and 51 years of age, a relative plateau is observed in which the BMI index remains constant above the value of $25 \mathrm{~kg} / \mathrm{m}^{2}$.

The strong point of this study is represented by the number of patients, 180, with a homogeneous distribution by age categories. However, when analyzing the points of maximum divergence in the graphs resulting from the data entry at those points, the number of patients is reduced, as a result the graph may be corrupted in critical points by a small number of patients taken into account. One direction to follow would be to observe the maintenance of the same trend in the case of a larger value sample. Throughout the age range taken into account, 40-84 years, there is a relatively linear evolution between BMI and the number of teeth up to the age of 55 years. After the age of 55, the trend is divergent, inversely correlated between BMI and the number of teeth. This is a possible conflicting conclusion with some studies denoting the irrelevance of BMI with the number of teeth after the age of 60 years. Some studies identified in the literature show that the age of 75 years from which BMI is no longer relevant in relation to the number of teeth. It should be noted that within Group I, premenopause, there is a homogeneity in 
the evolution of BMI and the number of teeth with a record of disparity towards the end of the interval, when the number of teeth decreases and BMI increases. In group II, postmenopause, there was a graphic heterogeneity, the age of 65 being the cutoff point from which BMI and the number of teeth change their correlation from positive to negative. A future direction would be to correlate this information with the level of education, individual and/ or family income, hormonal medication, eating and/or smoking habits, cardiovascular diseases, diabetes, neuropathies. These have been identified as having an impact on oral health but also on the control of menopausal disorders. From a dental point of view, in correlation with the age of menopause, parameters such as the bacterial plaque index, the depth of the gingival sacs, as well as the evaluation of bone density based on indices identi-

Conflict of interest: none declared

Financial support: none declared

\section{$\overline{\text { REFERENCES }}$}

1. McKinlay SM, Brambilla DJ, Posner JG. The normal menopause transition. Maturitas. 1992 Jan;14(2):103-15.

2. American Academy on Pediatric Dentistry Council on Clinical Affairs Committee on the Adolescent. Guideline on oral health care for the pregnant adolescent. Pediatr Dent. 2008-2009;30(7 Suppl):102-6.

3. Suresh L, Radfar L. Pregnancy and lactation. Oral Surg Oral Med Oral Pathol Oral Radiol Endod. 2004 Jun;97(6):672-82.

4. Migliaccio S, Greco EA, Fornari R, Donini LM, Lenzi A. Is obesity in women protective against osteoporosis? Diabetes Metab Syndr Obes. 2011;4:273-82.

5. Jeon YK, Lee JG, Kim SS, Kim BH, Kim SJ, Kim YK, Kim IJ. Association between bone mineral density and metabolic syndrome in pre- and postmenopausal women. Endocr J. 2011;58(2):87-93.

6. Hwang DK, Choi HJ. The relationship between low bone mass and metabolic syndrome in Korean women. Osteoporos Int. 2010 Mar;21(3):425-31.

7. Koh JM, Khang YH, Jung CH, Bae S, Kim DJ, Chung YE, Kim GS. Higher circulating hsCRP levels are associated with lower bone mineral density in healthy pre- and postmenopausal women: evidence for a link between systemic inflammation and osteoporosis. Osteoporos Int. 2005 Oct;16(10):1263-71.

8. Krall EA, Dawson-Hughes B, Papas A, Garcia RI. Tooth loss and skeletal bone density in healthy postmenopausal women. Osteoporos Int. 1994 Mar;4(2):104-9.

9. Tezal M, Wactawski-Wende J, Grossi SG, Ho AW, Dunford R, Genco RJ. The relationship between bone mineral density and fiable on dental radiography can also be added. If these factors were correlated with effective hormonal determinations, then the impact of these correlations on public health could be significant both by identifying risk factors and by diagnostic and/or therapeutic indications.

\section{CONCLUSIONS}

Premenopausal women have more teeth than postmenopausal women on average, both groups of patients being categorized as overweight.

Until the age of 55, there is a linear relationship between BMI and the number of teeth across the age range studied, which is 40 to 84 years. The trend is inversely correlated between BMI and the number of teeth after the age of 55 .

periodontitis in postmenopausal women. J Periodontol. 2000 Sep;71(9):1492-8.

10. Liljestrand JM, Havulinna AS, Paju S, Männistö S, Salomaa V, Pussinen PJ. Missing Teeth Predict Incident Cardiovascular Events, Diabetes, and Death. J Dent Res. 2015 Aug;94(8):1055-62.

11. López-Gómez SA, Villalobos-Rodelo JJ, Ávila-Burgos L, CasanovaRosado JF, Vallejos-Sánchez AA, Lucas-Rincón SE, Patiño-Marín $\mathrm{N}$, Medina-Solís CE. Relationship between premature loss of primary teeth with oral hygiene, consumption of soft drinks, dental care, and previous caries experience. Sci Rep. 2016 Feb 26;6:21147.

12. Hu HY, Lee YL, Lin SY, Chou YC, Chung D, Huang N, Chou YJ, Wu CY. Association Between Tooth Loss, Body Mass Index, and All-Cause Mortality Among Elderly Patients in Taiwan. Medicine (Baltimore). 2015 Sep;94(39):e1543.

13. Bole C, Wactawski-Wende J, Hovey KM, Genco RJ, Hausmann E. Clinical and community risk models of incident tooth loss in postmenopausal women from the Buffalo Osteo Perio Study. Community Dent Oral Epidemiol. 2010 Dec;38(6):487-97.

14. Oikarinen R, Syrjälä AM, Komulainen K, Knuuttila M, Ruoppi $P$, Hartikainen S, Sulkava R, Ylöstalo P. Body mass index and periodontal infection in a sample of non-smoking older individuals. Oral Dis. 2014 Apr;20(3):e25-30. 